\title{
OFDM with Index Modulation Assisted by Multiple Amplify-and-Forward Relays
}

\author{
Guo Sheng, Shuping Dang, Member, IEEE, Zhenrong Zhang, Member, IEEE, Enis Kocan, Member, IEEE and \\ Milica Pejanovic-Djurisic, Member, IEEE
}

\begin{abstract}
A multi-relay assisted orthogonal frequency division multiplexing (OFDM) system with index modulation (OFDM-IM) is proposed in this letter, assuming that amplify-and-forward (AF) relaying protocol is adopted at relays. Two commonly used AF protocols are considered: fixed-gain (FG) AF and variable-gain (VG) AF relaying protocols. To utilize relays in an efficient manner, we also employ two multi-carrier relay selection schemes termed bulk and per-subcarrier (PS) relay selection in the proposed system. We analyze the outage performance of the proposed system and derive closed-form expressions of the average outage probabilities (AOPs) for all cases with different AF relaying protocols and multi-carrier relay selection schemes. In addition, we obtain the asymptotic expressions of AOPs in the high signal-to-noise ratio (SNR) region, by which it is proven that the full diversity gain equaling the number of relays is attainable.
\end{abstract}

Index Terms-OFDM with index modulation, amplify-andforward relaying, multi-carrier relay selection, diversity gain.

\section{INTRODUCTION}

Novel communication paradigms, e.g., machine-type communications (MTC) and the Internet of Things (IoT), have brought new challenges to wireless communications systems. As a result, not only the exponential growth in data traffic, but also the considerable growth in the number of heterogeneous communication nodes shall be dealt with in next generation networks with innovative solutions starting from the physical layer, up to the complete network architecture [1], [2]. To support such high and heterogeneous transmission requirements, orthogonal frequency division multiplexing with index modulation (OFDM-IM), as a physical layer based solution, was proposed in [3] and it rapidly drew the attention from academia and industry [4]. Different from the classic

This work was supported by the National Natural Science Foundation of China under Grant 61661004, State Key Laboratory of Advanced Optical Communication Systems Networks and Guangxi Science Foundation (2017GXNSFAA198227).

G. Sheng is with the Department of Communication Engineering, Zhejiang Post and Telecommunication College, Shaoxing 312016, China (e-mail: sg@zptc.cn).

S. Dang was with the R\&D Center, Guangxi Huanan Communication Co., Ltd., Nanning 530007, China when participating in this work, and is now with Computer, Electrical and Mathematical Science and Engineering Division, King Abdullah University of Science and Technology (KAUST), Thuwal 23955-6900, Kingdom of Saudi Arabia (e-mail: shuping.dang@kaust.edu.sa)

Z.R. Zhang is with the School of Computer, Electronics and Information, Guangxi University, and Guangxi Key Laboratory of Multimedia Communications and Network Technology, Nanning 530004, China (e-mail: zzr76@gxu.edu.cn).

E. Kocan and M. Pejanovic-Djurisic are with University of Montenegro, Faculty of Electrical Engineering, Podgorica 81000, Montenegro (e-mail: \{enisk, milica\}@ucg.ac.me)
OFDM and other modulation schemes that only exploit the amplitude and phase dimensions, OFDM-IM introduces a new modulation dimension termed the index dimension and 'piggybacks' additional information by the indices of subcarriers or subcarrier activation patterns (SAPs). By properly configuring system setups, OFDM-IM is able to outperform the classic OFDM in terms of spectral efficiency [5].

However, compared to another rising index modulation scheme-spatial modulation (SM), OFDM-IM has a lower energy efficiency. To alleviate this shortcoming, decode-andforward (DF) cooperative relaying is introduced to enhance the transmission of OFDM-IM systems and harvest spatial diversity by relay selection [6], [7]. Although DF relaying is mathematically tractable, when it comes to practical realizations, some constraints may occur to hinder its implementation, especially in the IoT and MTC networks, where a large number of power-limited and/or complexity-limited nodes exist. Apart from DF cooperative relaying, amplifyand-forward (AF) relay selection has also been proved to be able to harvest diversity gain for generic cooperative systems with even lower complexity ${ }^{1}$ [8]-[10]. Therefore, to provide an easy-to-implement and practical approach to enhance the system reliability and harvest selection diversity for relay assisted OFDM-IM systems, we propose the multi-relay assisted OFDM-IM system, in which AF relaying protocol is adopted and multi-carrier relay selection is used to deploy all relays in an efficient manner. In particular, we consider two AF relaying strategies: fixed-gain (FG) $\mathrm{AF}$ and variable-gain (VG) AF relaying. Two commonly used multi-carrier relay selection schemes [11], termed the bulk and per-subcarrier (PS) relay selections are adopted, and the outage performance of the resultant systems is analyzed in this letter.

\section{System ModeL}

We consider a single source, $L$ AF relays (the set of AF relays is denoted as $\mathcal{L}=\{1,2, \ldots, L\}$ ) and a single destination, which constitute a commonplace two-hop cooperative network. In order to carry out relay selection, it is supposed that CSI is available at both source and destination in a slow fading environment. Based on the known CSI, by certain multi-carrier relay selection scheme, a subset of AF relays is selected to act as intermediate nodes between source and

\footnotetext{
${ }^{1}$ In particular, DF relaying is required to estimate the instantaneous channel state information (CSI), but this requirement can be relaxed in AF relaying. Moreover, there is no decoding/re-encoding module in AF relays to carry out received signal detection, and thereby a much simpler and faster forwarding processing is achievable by $\mathrm{AF}$ relaying.
} 
destination $^{2}$. We assume a scenario without direct communication link between source and destination. In addition, we suppose that the half-duplex forwarding protocol is accepted by all AF relays, and thereby two orthogonal time slots are indispensable for one complete transmission attempt. Besides, a sufficiently long cyclic prefix (CP) is inserted in the transmit OFDM block to yield $N$ independent subcarriers (the set of subcarriers is denoted as $\mathcal{N}=\{1,2, \ldots, N\}$ ).

Unlike the classic OFDM that uses all subcarriers to transfer data symbols, OFDM-IM keeps only part of the subcarriers as active, all depending on the incoming information bit stream. Namely, OFDM-IM separates a complete bit stream into two parts: the heading bit stream and the subsequent bit stream. The former is mapped to a SAP, and the latter is mapped to data symbols carried on active subcarriers. In this letter, all incoming bits are supposed to be equiprobable without loss of generality. As the kernel of OFDM-IM, in the following, we explain the mapping procedure. First, taking into account all available subcarriers in $\mathcal{N}$, we stipulate that an intact SAP is constituted by $N$ subcarrier activation states. $T$ out of $N$ subcarriers are afterword assigned to be activated and they create the activation subcarrier subset ${ }^{3} \mathcal{T}(k)$, where $k$ represents the index of SAP. Evidently, there are $K=\left(\begin{array}{l}N \\ T\end{array}\right)$ unique SAPs (the set of SAPs is denoted as $\mathcal{K}=\{1,2, \ldots, K\}$ ), which can be used to represent $B_{S}=\left\lfloor\log _{2}\left(\begin{array}{c}N \\ T\end{array}\right)\right\rfloor$ bits as the heading bit stream and $\lfloor\cdot\rfloor$ is the floor function. Meanwhile, as the numbers of SAPs in use must be the power of two because of the duality of a single bit, we have to shrink the full set $\mathcal{K}$ to the set of $\mathcal{U}$ with $U=2^{B_{S}}$ selected SAPs. Note that, there exist a variety of methods mapping bit sequences to $U$ SAPs, including the look-up table method, the combinatorial method, and adaptive methods [3], [13]-[15]. For simplicity, we adopt the look-up table method in this letter. Since different data symbols can be carried by different active subcarriers, $T$ $M$-ary amplitude-phase modulated (APM) data symbols are carried on these $T$ active subcarriers in order to enable their full exploitation and raise the spectral efficiency. The set of $M$-ary data symbols is denoted as $\mathcal{M}$. Obviously, we have $B_{M}=T \log _{2}(M)$, and the total transmission rate in bit per channel use (bpcu) is calculated by $B=B_{S}+B_{M}$.

To represent a unique SAP $k$ and $T$ data symbols borne on the $T$ active subcarriers, we generate a transmit OFDM block by an $N$-point inverse fast Fourier transform (IFFT) to be $\mathbf{x}(k)=\left[x\left(m_{1}, 1\right), x\left(m_{2}, 2\right), \ldots, x\left(m_{N}, N\right)\right]^{T} \in \mathbb{C}^{N \times 1}$, where $m_{n} \in \mathcal{M}$ denotes the index of data symbol carried on the $n$th subcarrier; the entry $x\left(m_{n}, n\right)$ of the transmit OFDM block $\mathbf{x}(k)$ is a normalized $M$-ary data symbol (i.e., $\left.x\left(m_{n}, n\right) x\left(m_{n}, n\right)^{*}=1\right)$, if the $n$th subcarrier is activated, or zero otherwise. The received signal on the $n$th active subcarrier at the $l$ th AF relay can be written as $y_{1}\left(m_{n}, n, l\right)=$ $\sqrt{P_{t} / T} h_{1}(n, l) x\left(m_{n}, n\right)+w_{1}(n, l)$, where $w_{1}(n, l)$ denotes

\footnotetext{
${ }^{2}$ Considering the limits on the system complexity and energy consumption at AF relay nodes, a centralized control mechanism can be employed to implement the relay selection schemes and broadcast the information regarding which relays shall forward on which subcarriers [12].

${ }^{3}$ The number of active subcarriers $T$ is a preset system parameter and associated with the well-known diversity-multiplexing trade-off. One should consider the system requirements and conditions when setting $T$, which are out of the scope of this letter. Interested readers might refer to [3].
}

complex additive white Gaussian noise (AWGN) sample with average noise power $N_{0} ; P_{t} / T$ is the uniform PS power; $h_{i}(n, l)$ represents the channel coefficient of the $n$th subcarrier forwarded the by the $l$ th AF relay over the $i$ th hop. As all channels are assumed to be slow and Rayleigh faded, the channel power gains $G_{i}(n, l)=\left|h_{i}(n, l)\right|^{2}$ are supposed to be independent and identically distributed (i.i.d.) random variables and exponentially distributed with mean $\mu_{i}$ for the $i$ th hop. Therefore, we can express the probability density function (PDF) of channel power gains as $f_{G_{i}}(\zeta)=\exp \left(-\zeta / \mu_{i}\right) / \mu_{i}$ and corresponding cumulative distribution function (CDF) as $F_{G_{i}}(\zeta)=1-\exp \left(-\zeta / \mu_{i}\right)$.

After receiving $y_{1}\left(m_{n}, n, l\right)$, the $l$ th selected AF relay simply amplifies the received signal and produces the retransmitted signal as $z\left(m_{n}, n, l\right)=\Gamma_{(\cdot)} y_{1}\left(m_{n}, n, l\right)$, where $\Gamma_{(\cdot)}$ is the amplification gain, which can be explicitly given for classic $\mathrm{FG} \mathrm{AF}$ and $\mathrm{VG} \mathrm{AF}$ relaying strategies as $\Gamma_{F}=$ $\sqrt{P_{t} /\left(\mu_{1} P_{t}+T N_{0}\right)}$ and $\Gamma_{V}=\sqrt{P_{t} /\left(P_{t} G_{1}(n, l)+T N_{0}\right)}$, respectively [16]. Alike the circumstance at relays, the signal received on the $n$th subcarrier at the destination, which is forwarded by the $l$ th relay is given by $y_{2}\left(m_{n}, n, l\right)=$ $h_{2}(n, l) z\left(m_{n}, n\right)+w_{2}(n)$, where $w_{2}(n)$ denotes the complex AWGN sample, which shares the identical statistical property as $w_{1}(n, l)$. Consequently, the end-to-end SNR for the $n$th active subcarrier forwarded by the $l$ th relay at the destination can be expressed by a general form as $\gamma_{(\cdot)}(n, l)=$ $\frac{\Gamma_{(\cdot)}^{2} G_{1}(n, l) G_{2}(n, l) P_{t}}{\left(\Gamma^{2}{ }_{(\cdot)} G_{2}(n, l)+1\right) T N_{0}}$, which can also be explicitly expressed for FG AF and VG AF relaying schemes by replacing $\Gamma_{(\cdot)}$ with $\Gamma_{F}$ and $\Gamma_{V}$ respectively: $\gamma_{F}(n, l)=\frac{P_{t}^{2} G_{1}(n, l) G_{2}(n, l)}{\left(\mu_{1} P_{t}+P_{t} G_{2}(n, l)+T N_{0}\right) T N_{0}}$ and $\gamma_{V}(n, l)=\frac{P_{t}^{2} G_{1}(n, l) G_{2}(n, l)}{\left(P_{t} G_{1}(n, l)+P_{t} G_{2}(n, l)+T N_{0}\right) T N_{0}}$.

For relay selection, two fundamental and widely implemented multi-carrier relay selection schemes are considered in this letter, which are the bulk and PS relay selection schemes [6]. When employing bulk relay selection, all active subcarriers are received and forwarded by only one single relay, whereas PS relay selection assigns multiple (up to $T$ ) relays to forward different active subcarriers on a PS basis. The selection criteria of both multi-carrier relay selection schemes can be modified for multi-relay assisted OFDM-IM systems to be

$$
\left\{\begin{array}{l}
\mathcal{L}_{\text {bulk }}=\arg \max _{l \in \mathcal{L}}\left\{\min _{n \in \mathcal{N}}\left\{\gamma_{(\cdot)}(n, l)\right\}\right\} \\
\mathcal{L}_{\text {ps }}=\bigcup_{n \in \mathcal{N}}\left\{\arg \max _{l \in \mathcal{L}}\left\{\gamma_{(\cdot)}(n, l)\right\}\right\}
\end{array}\right.
$$

\section{Outage Performance Analysis}

\section{A. Preliminaries}

To investigate the outage performance of multi-relay assisted OFDM-IM systems in a holistic manner, we provide a system-level interpretation of the outage event considering the multi-carrier, multi-hop and multi-relay scenarios [17]:

Remark 1: An outage event occurs once the end-to-end SNR of any active subcarrier falls below an outage threshold s. This gives the conditional outage probability (OP) on the $k$ th SAP after performing multi-carrier relay selection by

$$
P_{o}(s \mid k)=\mathbb{P}\left\{\bigcup_{i=1,2}\left\{\bigcup_{n \in \mathcal{T}(k)}\left\{\gamma_{(\cdot)}(l(n), n)<s\right\}\right\}\right\},
$$


where $\mathbb{P}\{\cdot\}$ represents the probability of the random event enclosed and $l(n)$ stands for the index of relay selected to forward the $n$th subcarrier, given $n \in \mathcal{T}(k)$. We herewith express the average OP (AOP) by $\bar{P}_{o(\cdot)}(s)=\underset{k \in \mathcal{U}}{\mathbb{E}}\left\{P_{o(\cdot)}(s \mid k)\right\}$, where $\mathbb{E}\{\cdot\}$ represents the mean of the random variable enclosed. AOP is of high importance and can be used as an essential as well as handy metric for assessing the reliability of multicarrier communication systems. We hereby adopt AOP as the performance assessment metric in the following analysis.

To derive the AOP defined above, we first have the a priori conditional OP given by $\Phi_{(\cdot)}(s \mid k)=\mathbb{P}\left\{\gamma_{(\cdot)}(n, l)<s\right\}$ for both FG AF and VG AF relaying in (3) at the top of the next page [16], where $K_{\nu}(\cdot)$ denotes the $\nu$ th order modified Bessel function of the second kind. From (3), we can observe that the condition on SAP $k$ has been removed, since all subcarriers are i.i.d. and the number of active subcarriers is fixed to $T$. Consequently, all SAPs are statistically equivalent. By this property, we thereupon deduce $\Phi_{(\cdot)}(s)=\underset{k \in \mathcal{U}}{\mathbb{E}}\left\{\Phi_{(\cdot)}(s \mid k)\right\}=\Phi_{(\cdot)}(s \mid k)$ and $P_{o(\cdot)}(s)=\underset{k \in \mathcal{U}}{\mathbb{E}}\left\{P_{o(\cdot)}(s \mid k)\right\}=P_{o(\cdot)}(s \mid k)$, by which it is clear that the outage performance of the proposed OFDM-IM system is dependent on the number of active subcarriers $T$, rather than the number of total subcarriers $N$.

\section{B. Bulk Relay Selection}

According to (1) and fundamentals of order statistics, it is straightforward to express the a posteriori OP conditioning on bulk relay selection by ${ }^{4}$

$$
P_{o(\cdot)}(s)=\left[1-\left(1-\Phi_{(\cdot)}(s)\right)^{T}\right]^{L},
$$

which is also the AOP when bulk relay selection is employed in accordance with what we derived in Section III-A.

To scrutinize the relation among the outage performance and key system parameters and configurations, we resort to the power series expansion in (4) at high SNR (i.e., let $P_{t} \rightarrow \infty$ ) and derive the asymptotic expression (the leading order term) of (4) for both FG AF and VG AF relaying schemes to be

$$
\left\{\begin{array}{l}
P_{o F}(s) \sim\left(\frac{s N_{0} T^{2}}{\mu_{2} P_{t}} \log \left(\frac{\mu_{2} P_{t}}{s N_{0} T} \exp \left(1-2 \gamma+\frac{\mu_{2}}{\mu_{1}}\right)\right)\right)^{L} \\
P_{o V}(s) \sim\left[\frac{s N_{0} T^{2}}{P_{t}}\left(\frac{1}{\mu_{1}}+\frac{1}{\mu_{2}}\right)\right]^{L}
\end{array}\right.
$$

where $\gamma \approx 0.577$ is the Euler-Mascheroni constant.

\section{Per-Subcarrier Relay Selection}

The a posteriori OP, i.e., the AOP, conditioning on the PS relay selection can be likewise obtained according to (1) as

$$
P_{o(\cdot)}(s)=1-\left[1-\left(\Phi_{(\cdot)}(s)\right)^{L}\right]^{T} \text {. }
$$

One more time, power series expansion is performed at $P_{t} \rightarrow$ $\infty$ to yield the asymptotic expression of (6) at high SNR for both FG AF and VG AF relaying to be

$$
\left\{\begin{array}{l}
P_{o F}(s) \sim T\left(\frac{s N_{0} T}{\mu_{2} P_{t}} \log \left(\frac{\mu_{2} P_{t}}{s N_{0} T} \exp \left(1-2 \gamma+\frac{\mu_{2}}{\mu_{1}}\right)\right)\right)^{L} \\
P_{o V}(s) \sim T\left[\frac{s N_{0} T}{P_{t}}\left(\frac{1}{\mu_{1}}+\frac{1}{\mu_{2}}\right)\right]^{L}
\end{array}\right.
$$

\footnotetext{
${ }^{4}$ The detailed derivation steps of (4) as well as (6) in the sequel can be found in [11] for a similar scenario of classic relayed OFDM systems.
}

From these derived asymptotic expressions given in (5) and (7), it is evident that the full diversity gain equaling the number of AF relays $L$ can be achieved for both AF relaying schemes when either bulk or PS relay selection is in use.

\section{Simulation Results and Discussion}

To substantiate the outage performance analysis presented in Section III, we carried out numerical simulations by Monte Carlo methods and present the simulation results with discussions in this section. We set up $N=8$ and normalize $s$, $N_{0}, \mu_{1}$ and $\mu_{2}$ so as to be general (i.e., $s=1, N_{0}=1$, $\mu_{1}=\mu_{2}=1$ ). The APM scheme adopted on all active subcarriers is binary phase-shift keying (BPSK). The simulation results of AOP versus the power ratio $P_{t} / N_{0}$ for various cases with different relay selection schemes, AF relaying protocols and configuration parameter sets $\{T, L\}$ (the numbers of active subcarriers and relays) are illustrated in Fig. 1.

From Fig. 1, several key observations are discussed as follows. Above all else, the derived outage performance expressions of the proposed multi-relay assisted OFDM-IM system given in (4) and (6) have been verified by the simulations provided. This is certified by excellent matching between simulation and analytical results. Just as important, with an increasing power ratio $P_{t} / N_{0}$, the asymptotic results approach the simulation results, thus verifying the asymptotic performance analysis. Besides, the simulation results confirm that the full diversity gain equaling the number of relays $L$ is attainable at high SNR. In addition, the outage performance superiority of PS relay selection over bulk relay selection can also be illustrated by comparing the simulation results denoted by black and blue markers, which is resulted by a higher coding gain via the multi-relay selection mechanism.

Furthermore, we illustrate the effects of different relaying protocols on the outage performance of relay assisted OFDMIM systems employing multi-carrier relay selections in Fig. 2 , given $\{T, L\}=\{2,2\}$. Apart from the classic FG AF and VG AF relaying schemes analyzed in this letter, we adopt the DF relaying as the comparison benchmark. The comparison among various relaying protocols well reflects the trade-off between system complexity and outage performance. The DF relaying that requires the full instantaneous CSI has the best outage performance and serves as a lower bound on AOP compared to AF relaying protocols. The FG AF relaying that requires the least CSI (only the statistical CSI for the first hop) has the worst performance. Therefore, in practical communication networks, which relaying scheme should be adopted depends on the channel unpredictability, limits on system device complexity and energy consumption as well as the required performance. It would be difficult to ascertain whether one outperforms the others in general.

\section{CONClusion}

In this letter we proposed a multi-relay assisted OFDM-IM, applying AF relaying and multi-carrier relay selection, as a solution with the significant potential to enhance the system reliability in new network paradigms, where a large number of simple, power-limited and/or complexity-limited nodes exist 


$$
\left\{\begin{array}{l}
\Phi_{F}(s \mid k)=1-2 \exp \left(-\frac{s N_{0} T}{\mu_{1} P_{t}}\right) \sqrt{\frac{s N_{0} T\left(\mu_{1} P_{t}+N_{0} T\right)}{\mu_{1} \mu_{2} P_{t}^{2}}} K_{1}\left(2 \sqrt{\frac{s N_{0} T\left(\mu_{1} P_{t}+N_{0} T\right)}{\mu_{1} \mu_{2} P_{t}^{2}}}\right) \\
\Phi_{V}(s \mid k)=1-2 \exp \left(-\frac{s N_{0} T}{P_{t}}\left(\frac{1}{\mu_{1}}+\frac{1}{\mu_{2}}\right)\right) \sqrt{\frac{s N_{0}^{2} T^{2}}{\mu_{1} P_{t}^{2}}\left(s+\frac{1}{\mu_{2}}\right)} K_{1}\left(2 \sqrt{\frac{s N_{0}^{2} T^{2}}{\mu_{1} P_{t}^{2}}\left(s+\frac{1}{\mu_{2}}\right)}\right)
\end{array}\right.
$$

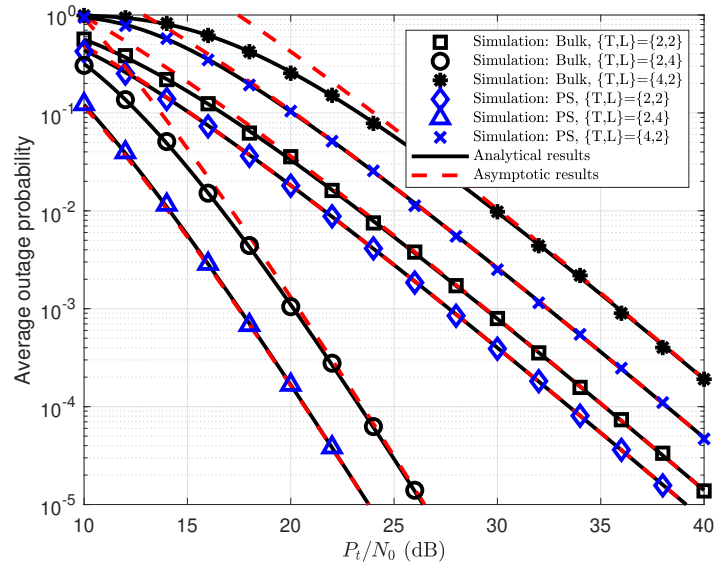

(a) FG AF relaying

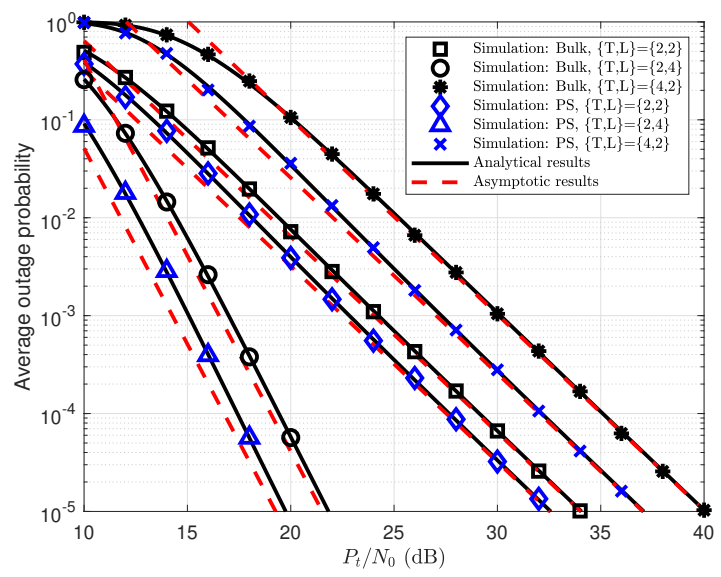

(b) VG AF relaying

Fig. 1: AOP vs. power ratio $P_{t} / N_{0}$ for different cases.

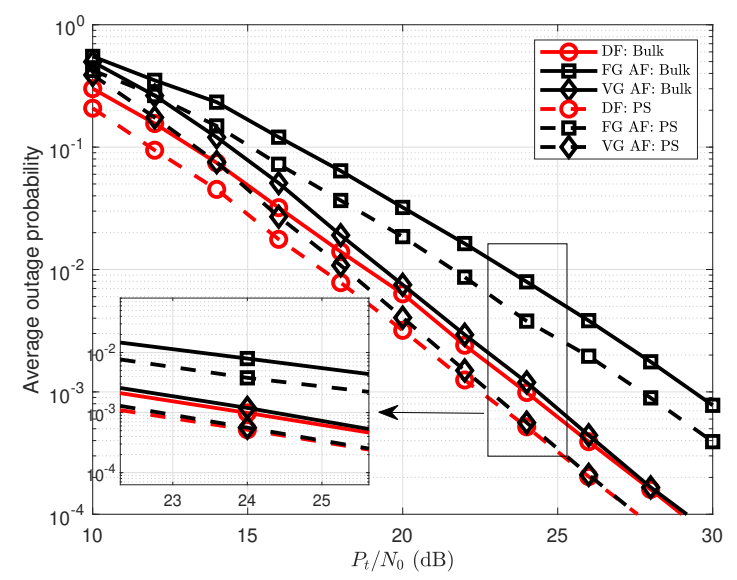

Fig. 2: Comparison of AOP among a variety of relaying protocols. (e.g., MTC networks and the IoT). We analyzed the outage performance of the proposed systems and corroborated all analytical results through numerical simulations.

\section{REFERENCES}

[1] J. G. Andrews, S. Buzzi, W. Choi, S. V. Hanly, A. Lozano, A. C. K. Soong, and J. C. Zhang, "What will 5G be?" IEEE Journal on Selected Areas in Communications, vol. 32, no. 6, pp. 1065-1082, June 2014.

[2] G. Chen, J. Tang, and J. P. Coon, "Optimal routing for multihop socialbased D2D communications in the internet of things," IEEE Internet of Things Journal, vol. 5, no. 3, pp. 1880-1889, June 2018.

[3] E. Basar, U. Aygolu, E. Panayırc1, and H. V. Poor, "Orthogonal frequency division multiplexing with index modulation," IEEE Transactions on Signal Processing, vol. 61, no. 22, pp. 5536-5549, Nov. 2013.

[4] X. Cheng, M. Zhang, M. Wen, and L. Yang, "Index modulation for 5G: Striving to do more with less," IEEE Wireless Communications, vol. 25, no. 2, pp. 126-132, Apr. 2018.

[5] M. Wen, X. Cheng, M. Ma, B. Jiao, and H. V. Poor, "On the achievable rate of OFDM with index modulation," IEEE Transactions on Signal Processing, vol. 64, no. 8, pp. 1919-1932, Apr. 2016.

[6] S. Dang, G. Chen, and J. P. Coon, "Outage performance of two-hop OFDM with index modulation and multi-carrier relay selections," IEEE Wireless Communications Letters, 2018.

[7] J. Crawford and Y. Ko, "Cooperative OFDM-IM relay networks with partial relay selection under imperfect CSI," IEEE Transactions on Vehicular Technology, 2018.

[8] G. Chen and J. A. Chambers, "Exact outage probability analysis for cooperative AF relay network with relay selection in presence of intercell interference," Electronics Letters, vol. 48, no. 21, pp. 1346-1347, Oct. 2012.

[9] M. A. Manna, G. Chen, and J. A. Chambers, "Outage probability analysis of cognitive relay network with four relay selection and endto-end performance with modified quasi-orthogonal space-time coding," IET Communications, vol. 8, no. 2, pp. 233-241, Jan. 2014.

[10] M. M. Eddaghel, U. N. Mannai, G. J. Chen, and J. A. Chambers, "Outage probability analysis of an amplify-and-forward cooperative communication system with multi-path channels and max-min relay selection," IET Communications, vol. 7, no. 5, pp. 408-416, Mar. 2013.

[11] L. Dai, B. Gui, and L. J. C. Jr., "Selective relaying in OFDM multihop cooperative networks," in Proc. IEEE WCNC, Kowloon, Hong Kong, China, Mar. 2007, pp. 963-968.

[12] T. Jamal and P. Mendes, "Cooperative relaying in user-centric networking under interference conditions," IEEE Communications Magazine, vol. 52 , no. 12 , pp. $18-24$, Dec. 2014

[13] M. Wen, Y. Zhang, J. Li, E. Basar, and F. Chen, "Equiprobable subcarrier activation method for OFDM with index modulation," IEEE Communications Letters, vol. 20, no. 12, pp. 2386-2389, Dec. 2016.

[14] S. Dang, J. P. Coon, and G. Chen, "Adaptive OFDM with index modulation for two-hop relay-assisted networks," IEEE Transactions on Wireless Communications, vol. 17, no. 3, pp. 1923-1936, Mar. 2018.

[15] S. Dang, G. Chen, and J. P. Coon, "Lexicographic codebook design for OFDM with index modulation," IEEE Transactions on Wireless Communications, 2018.

[16] D. E. Simmons and J. P. Coon, "Two-way OFDM-based nonlinear amplify-and-forward relay systems," IEEE Transactions on Vehicular Technology, vol. 65, no. 5, pp. 3808-3812, May 2016.

[17] W. Yang and Y. Cai, "On the performance of the block-based selective OFDM decode-and-forward relaying scheme for 4G mobile communication systems," Journal of Communications and Networks, vol. 13, no. 1, pp. 56-62, Feb. 2011. 